\title{
Mejora de la planificación temporal y adaptación de la metodología docente de la asignatura de IDEs del grado de Ingeniería Geomática mediante estadísticos de encuestas anónimas de horas dedicadas
}

\section{Martinez-Llario Jose ${ }^{\mathrm{a}}$, Coll Eloina}

${ }^{a}$ Departamento de Ingeniería Cartográfica, Geodesia y Fotogrametría, Universitat Politècnica de València, jomarlla@cgf.upv.es, ecoll@cgf.upv.es

\begin{abstract}
In this paper, a teaching methodology adapted to support a very flexible way of delivery times of the practical contents of a subject.

The aim is to give the student the possibility of using many more hours than the average in a given module in order to avoid sending incomplete tasks or abandoning the subject.

To do this, a series of surveys are used and with its statisticians the schedule of the subject is designed for the next course.

In addition, the methodology is adapted with reverse teaching and principally all the contents are adapted to give the possibility to the student to finish any task outside teaching hours.
\end{abstract}

Keywords: flip teaching, geomatics, polls

\footnotetext{
Resumen

En este artículo presentamos una metodología docente adaptada para soportar tiempos de entrega de los contenidos prácticas de una asignatura de forma muy flexible.

Se busca dar la posibilidad al alumno de utilizar muchas más horas que la media en un determinado módulo para así evitar el envío de tareas incompletas o abandono de la asignatura.

Para ello, se utilizan una serie de encuestas y con sus estadísticos se diseña el cronograma de la asignatura para el curso siguiente.

Además, se adapta la metodología con docencia inversa y principalmente se adaptan todos los contenidos para dar la posibilidad al alumno de finalizar cualquier tarea fuera de horario docente.
}

Palabras clave: gestión del tiempo, docencia inversa, encuesta, planificación temporal, geomática 
Mejora de la planificación temporal y adaptación de la metodología docente de la asignatura de IDEs del grado de Ingeniería Geomática mediante estadísticos de encuestas anónimas de horas dedicadas

\section{Introducción}

La metodología docente mostrada en este artículo se aplica desde hace cinco años en la asignatura de Infraestructuras de Datos Espaciales (IDEs) en el grado de Ingeniería Geomática de la ETSIGCT de la UPV. Es una asignatura de 6 créditos, dividida en 50\% prácticas de laboratorio y $50 \%$ de teoría.

El tiempo necesario para el desarrollo de los contenidos prácticos de esta asignatura requieren de unas habilidades que dependen mucho de las destrezas informáticas de cada alumno, y también de los problemas técnicos que se pueden encontrar en su realización y que pueden ser diferentes para cada alumno, ya que cada uno trabaja con diferentes datos.

El primer año de la asignatura el profesorado se encontró con los problemas mencionados en el párrafo anterior, y es que el tiempo necesario para la realización de los módulos de prácticas era muy variado en función de cada alumno, debido a dos causas:

- La heterogeneidad en las destrezas de cada alumno en cuanto a la aplicación de determinados conocimientos relaciones con las ciencias de la computación.

- Los problemas encontrados en cada módulo de prácticas pueden ser muy diferentes para cada alumno.

Nos encontramos que un mismo módulo de prácticas, un alumno lo realizaba en 4 horas mientras que otro podía necesitar 12 horas para su finalización.

Además, especialmente para los alumnos que empleaban en un módulo muchas horas, no podían finalizarlo en el laboratorio y debían de acabarlo fuera de horas lectivas.

\section{Objetivos}

Los objetivos consisten en diseñar una metodología docente que sea flexible en cuanto al tiempo necesario para cada alumno en la realización de prácticas pero siguiendo una estructura común basada en datos empíricos de años anteriores.

Se busca dar la posibilidad al alumno de utilizar muchas más horas que la media en un determinado módulo para así evitar el envío de tareas incompletas o abandono de la asignatura.

Para ello debemos de:

- Averiguar el tiempo medio de forma empírica en la realización de las prácticas de cada módulo.

- Crear un sistema flexible en cuanto a las fechas de entrega de los trabajos basado en los estadísticos obtenidos anteriormente.

- Adaptar el material de la asignatura para soportar la posibilidad de finalizar las prácticas fuera del horario docente.

- Adaptar la metodología docente con el uso de herramientas de formación a distancia para dar soporte fuera de horario docente.

(c) EY-NC-ND 2018, Universitat Politècnica de València

Congreso IN-RED (2018) 


\section{Desarrollo}

\subsection{Cálculo del tiempo empleado en cada módulo}

Para el cálculo del tiempo se empleó la herramienta de Sondeos de PoliformaT (realización de encuestas anónimas), cada alumno al finalizar la entrega de las prácticas de un módulo (la asignatura consta de 11 módulos) realiza una encuesta anónima (figura 1).

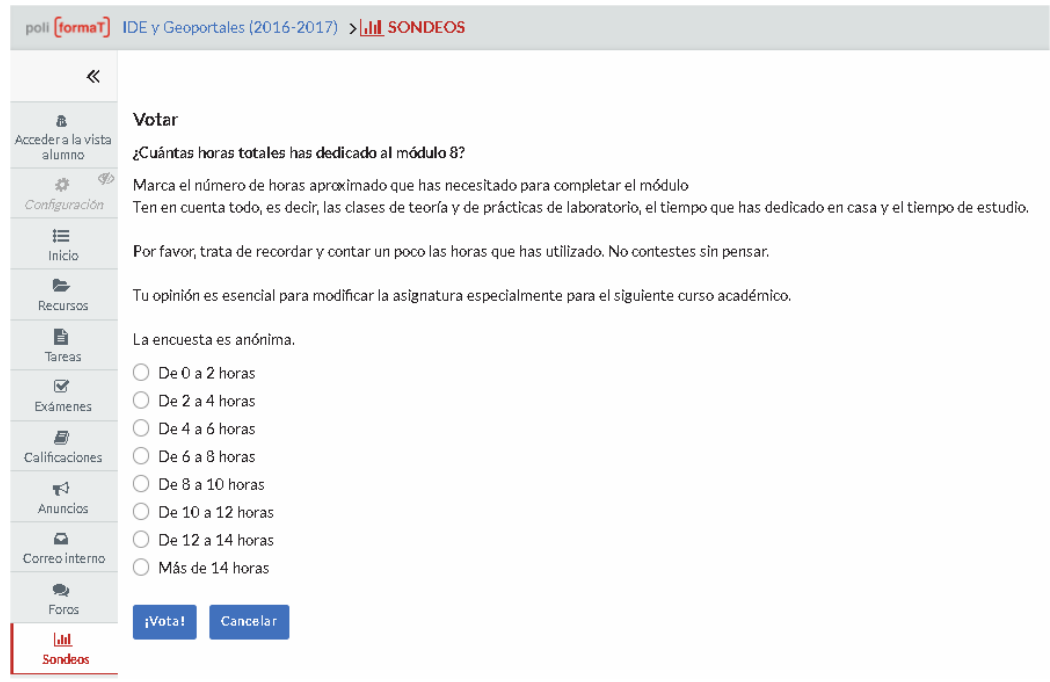

Fig. 1 Encuesta (sondeo en PoliformaT) anónima de un módulo

Los rangos de horas en la encuesta son amplios (2 horas) para facilitar la respuesta. Al finalizar el curso académico se estudian las encuestas y se obtienen los estadísticos para cada uno de los módulos.

En la figura 2 se puede ver como la moda de tiempo empleado en la finalización del módulo 2 corresponde sobre 9 horas (31\% de los alumnos), aunque un 15\% de los alumnos han necesitado más de 14 horas para su finalización lo cual indica la disparidad de las horas empleadas por los alumnos debido a las causas comentadas en el apartado anterior.

Se reduce en gran medida la posibilidad de que un alumno envíe el módulo sin completar, ya que se ha observado que cuando un alumno se encuentra con problemas en un módulo y requiere más horas de las normales para su realización prefiere dedicarle más horas aunque sea fuera de las horas lectivas y finalizar completamente el módulo. 
Mejora de la planificación temporal y adaptación de la metodología docente de la asignatura de IDEs del grado de Ingeniería Geomática mediante estadísticos de encuestas anónimas de horas dedicadas

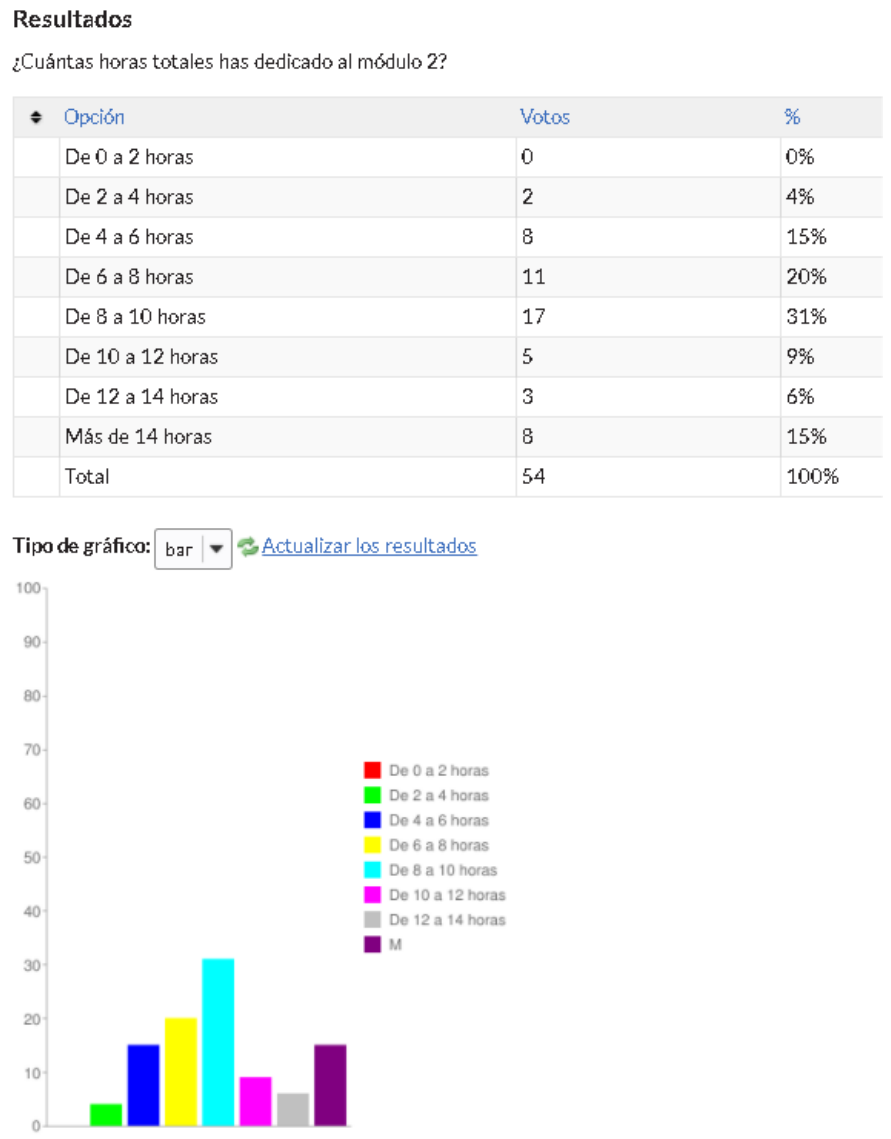

Fig. 2 Resultado al finalizar el curso de las encuestas de un módulo

\subsection{Sistema flexible de entrega}

Basado en los datos obtenidos de las encuestas se elabora un cronograma de rangos flexibles (entendiendo por rango flexible que el tiempo desde la apertura y a la entrega máxima permitida es fácilmente el doble o el triple del tiempo medio empleado por todos los alumnos del curso anterior) para la realización de cada módulo. La figura 3, muestra el cronograma que se ha realizado para el curso 2017-2018. La figura 2, mostraba el tempo medio empleado por un módulo.

Se puede ver como por ejemplo para el módulo 6, se da un total de 6 semanas para su realización. El alumno que emplee un número de horas medio (según los datos de las encuestas del año anterior) se moverá en la franja verde-amarilla.

Un alumno puede entregar el módulo 6 desde la primera semana de octubre hasta la tercera semana de noviembre como máximo.

Para los últimos módulos se alarga la fecha de entrega máxima hasta una semana antes de la entrega de las actas.

(cc) EY-NC-ND 2018, Universitat Politècnica de València 
Las fechas de entrega y duración de los módulos quedan plasmados en PoliformaT mediante las tareas cuyas fechas de apertura y entrega siguen de forma rigurosa el cronograma.
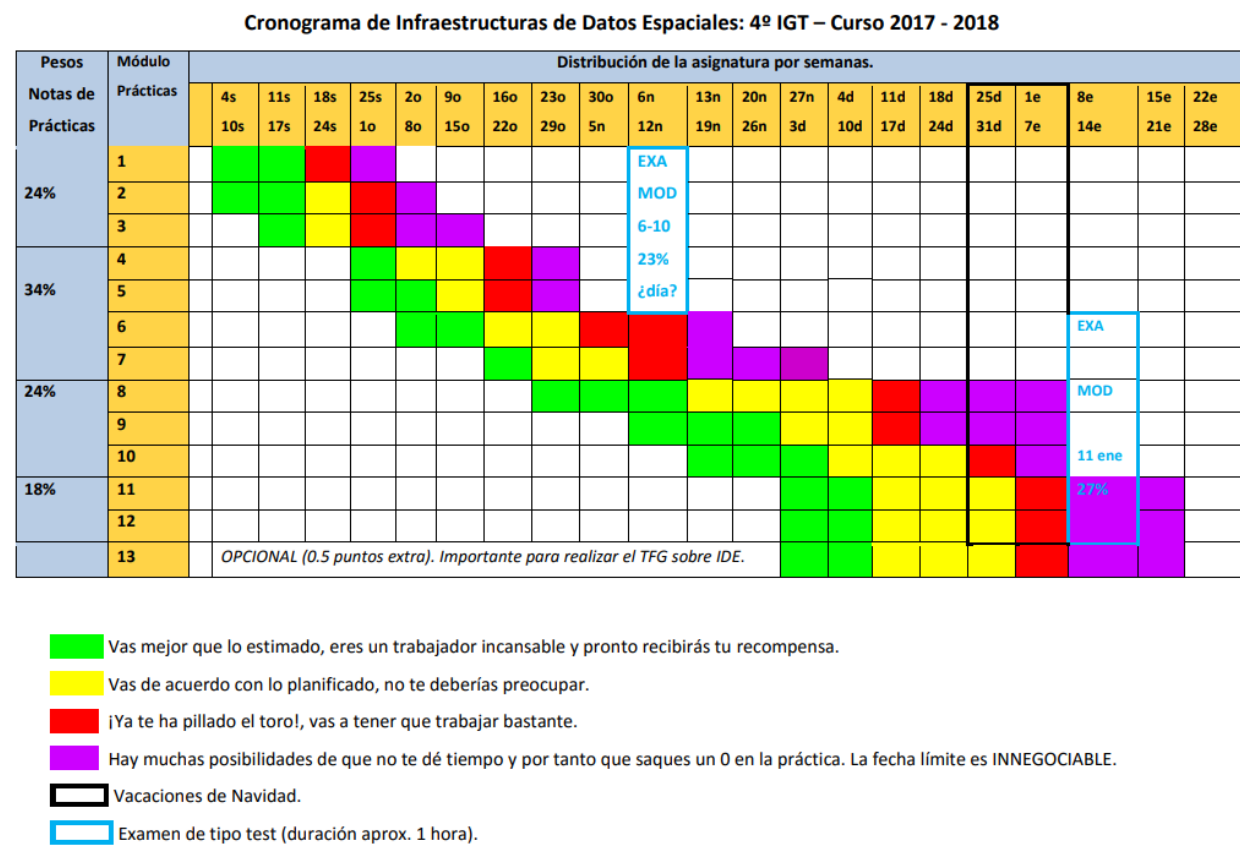

Fig. 3 Cronograma realizado a partir de las encuestas del curso del año anterior

\subsection{Adaptación del material docente}

Como se ha comentado anteriormente se debe dar la posibilidad al alumno de que finalice o realice cualquier tarea fuera del horario docente, así pues ha sido necesario rediseñar todo el material docente de las prácticas de la asignatura para que de forma autónoma un alumno puede trabajar fuera del laboratorio y completar parte de las prácticas. Se ha realizado una redacción y guiado minucioso de todas las prácticas.

Hay que comentar que cada alumno trabaja con datos y cartografía diferente y se evita de esta forma en gran medida que se copien las prácticas al tener la posibilidad de trabajar sin la supervisión del profesor.

Queremos mencionar que según los cálculos de las encuestas, aproximadamente los alumnos necesitan un 20-60\% de dedicación horaria fuera de laboratorio para finalizar todos los módulos.

\subsection{Herramientas de formación a distancia}

Se han incluido foros en PoliformaT para facilitar la respuesta del profesor o de otros compañeros cuando un alumno está realizando una práctica fuera de horario docente.

(c) EY-NC-ND 2018, Universitat Politècnica de València 
Mejora de la planificación temporal y adaptación de la metodología docente de la asignatura de IDEs del grado de Ingeniería Geomática mediante estadísticos de encuestas anónimas de horas dedicadas

También, se han incluido un gran número de vídeos screen cast y se ha creado una máquina virtual para que el alumno trabaje en el laboratorio con todo el software y una vez acabe la práctica se pueda llevar todo el trabajo realizado para su continuación si es necesario fuera del laboratorio.

\section{Resultados y conclusiones}

Se ha creado un sistema de encuestas, adaptado el material docente para que el alumno pueda finalizar las prácticas fuera de horario docente si es necesario y utiliza algunas herramientas de formación a distancia como la utilización de foros, vídeos screen cast, etc.

Hemos adaptado los tiempos de entrega de las tareas como aparece en la figura 4 permitiendo una flexibilidad que contemple la disparidad en horas que necesita cada alumno. Además en dicha figura se puede ver como prácticamente todos alumnos han entregado todas las prácticas.

\begin{tabular}{|c|c|c|c|c|}
\hline Título de la Tarea & Estado & Abierto & Entregạ & Entregados \\
\hline Prácticas módulo01 & Cerrado & 04-sep-2017 0:00 & 01-oct-2017 23:55 & $\underline{33 / 32}$ \\
\hline Prácticas módulo02 & Cerrado & $04-$ sер-2017 0:00 & 08 -oct-2017 23:55 & $\underline{31 / 28}$ \\
\hline Prácticas módulo 03 & Cerrado & $11-$ sep-2017 0:00 & 15 -oct-2017 23:55 & $\underline{32 / 30}$ \\
\hline Prácticas módulo04 & Cerrado & 25 -sер-2017 0:00 & 29 -oct-2017 23:55 & $\underline{34 / 30}$ \\
\hline Prácticas módulo05 & Cerrado & $25-$ sер-2017 0:00 & 29 -oct-2017 23:55 & $\underline{29 / 27}$ \\
\hline Prácticas módulo 06 & Cerrado & 02-oct-2017 0:00 & 26-nov-2017 23:55 & $\underline{33 / 31}$ \\
\hline SPácticas módulo 07 & Cerrado & 16 -oct-2017 0:00 & 10-dic-2017 23:55 & $\underline{29 / 27}$ \\
\hline Prácticas módulo 08 & Cerrado & $23-$ act-2017 0:00 & 07-ene-2018 23:55 & $\underline{34 / 33}$ \\
\hline Prácticas módulo09 & Cerrado & 06-nov-2017 0:00 & 07-ene-2018 23:55 & $\underline{33 / 33}$ \\
\hline Prácticas módulo 10 & Cerrado & $13-$ nov-2017 0:00 & 07-ene-2018 23:55 & $\underline{34 / 29}$ \\
\hline Prácticas módulo 11 & Cerrado & $27-n o v-20170: 00$ & 21-ene-2018 23:55 & $\underline{30 / 30}$ \\
\hline Prácticas módulo 12 & Cerrado & 27-nov-2017 0:00 & 21-ene-2018 23:55 & $\underline{26 / 22}$ \\
\hline
\end{tabular}

Fig. 4 Prácticas entregadas. Fechas adaptadas al cronograma de las encuestas

La metodología docente requiere un esfuerzo extra del profesor, debido a que es necesario la elaboración de un material que permita el alumno finalizar las tareas fuera de laboratorio si es necesario. Además, lo normal es que en el laboratorio los alumnos estén realizando prácticas de hasta 4 módulos diferentes y el profesor debe estar centrado para resolver todas las dudas de forma ágil.

Se ha observado que esta metodología evita abandonos en la asignatura y aumenta la tasa de rendimiento. Como conclusión podemos decir que un alumno prefiere si se le da la suficiente flexibilidad y lo necesita de forma puntual (al encontrarse con dificultades extra), dedicar muchas más horas que la media para finalizar un módulo que dejarlo sin entregar o realizarlo de forma no correcta.

También se ha comentado con los propios alumnos que el sistema les produce meno ansiedad y estrés que las entrega de prácticas fija o la obligación de finalización de una práctica en horario lectivo.

(cc) EY-NC-ND 2018, Universitat Politècnica de València

Congreso IN-RED (2018) 
Por último, decir que las encuestas docentes de la asignatura y también las encuestas de docencia inversa, corroboran que la metodología docente es del agrado de los alumnos.

\section{Referencias}

Barrera, A.G. (2013). El aula inversa: cambiando la respuesta a las necesidades de los estudiantes. Avances en Supervisión Educativa.

López Rodríguez, D., et al. (2016). Elaboración de material para la realización de experiencias de clase inversa (flipped classroom). ISBN 978-84-617-5129-7, págs. 16651679.

Polanco, A. (2005). “La motivación en los estudiantes universitarios”. Revista Electrónica "Actualidades Investigativas en Educación. Vol. 5, núm. 2, pp. 1-13

Salinas, J. (2004). Innovación docente y uso de las TIC en la enseñanza universitaria. Revista de Universidad y Sociedad del Conocimiento (RUSC). [artículo en línea]. UOC. Vol. $1, \mathrm{n}^{\mathrm{o}} 1$. 